\title{
The effect of transplantation of bone marrow cells induced by the contact with thymus-derived multipotent stromal cells on the immune system of mice, regenerating after cyclophosphamide treatment
}

\author{
Demchenko D. L. \\ State Institute of Genetic and Regenerative Medicine National Academy of Medical Sciences of Ukraine, Kyiv, Ukraine \\ e-mail: chekhdariia@gmail.com
}

\section{ABSTRACT}

The effect of transplantation of syngeneic bone marrow cells (BMCS) after their contact in vitro with thymus-derived multipotent stromal cells (MSCs) for regeneration of damaged by cyclophosphamide immune system of mice was studied.

MATERIALS AND METHODS. MSCs were obtained from C57BL/6 mice's thymus by explants method. BMCs were obtained by flushing the femurs. BMCs were induced for 2 hours on the monolayer of thymus-derived MSCs. The immune deficiency of mice was modelled using cyclophosphamide injection. After that, cell transplantation was performed and the state of the immune system was assessed. The number of erythrocytes, hematocrit, hemoglobin concentration in the peripheral blood; the phases of the cell cycle and apoptosis of mesenteric lymph node cells were determined. The amount of antibody-producing cells in the spleen and the delayed hypersensitivity response was determined. The study of proliferative and cytotoxic activity of natural killer lymphocytes, the analysis of phagocytosis, spontaneous and induced bactericidal activity of peritoneal macrophages were performed.

RESULTS. It was shown that unlike intact bone marrow cells, BMCs induced by thymus-derived MSCs provided increased spontaneous proliferative activity of lymphocytes with a decrease in the number of lymph node cells in $G_{d} G_{1}$ phase by $6.2 \%$ and an increase the number of lymphocytes in $S+G_{2} / M$ phase by $28 \%$ in comparison with the group of mice treated with cyclophosphamide, as well as the recovery of cellularity of the bone marrow, lymph nodes and spleen. At the same time in the lymph nodes, the number of cells in the apoptosis increased. BMCs induced by MSCs showed a pronounced negative effect on natural cytotoxicity, reducing its rates by 3 times compared with the group of cyclophosphamide-treated mice, and on adaptive immunity: the rates of delayed hypersensitivity response decreased by 1.7 times, the number of antibody-producing cells by 1.8 times. Red blood cell regeneration was stimulated by intact BMCs, which was manifested by the normalization of hematocrit and hemoglobin and an increase in the number of reticulocytes in the blood by 2.2 times compared with the group of mice treated with cyclophosphamide.

CONCLUSION. Transplanted BMCs improve erythropoiesis in mice after cyclophosphamide treatment, and BMCs, previously induced by thymusderived MSCs, lose this ability. BMCs after co-culture are strongly activated to impact on the immune system, which is most likely due to the effect of contact interaction with thymus-derived MSCs, effectively impact on hematopoietic cells and possess immunomodulatory properties.

KEY WORDS: hematopoietic bone marrow cells; thymus-derived multipotent stromal cells; regeneration of the immune system; cyclophosphamide-induced immunodeficiency 
The transplantation of bone marrow cells containing hematopoietic stem cells (HSCs) is used to regenerate the immune and hematopoietic systems. Historically, bone marrow transplantation was the first method of HSCs transplantation. HSCs are progenitor cells, which are mainly located in the specialized «niches» of the bone marrow in contact with mesenchymal multipotent stromal cells (MSCs) [1]. The effect of microenvironment provides the implementation of self-renewal, multipotency and proliferation programs in HSCs [2]. This allows to withdraw the required number of cells in the differentiation in certain directions, while maintaining a constant number of HSCs required for normal hematopoiesis. Multipotent stromal cells are the basis of bone marrow niches of HSCs and largely determine the realization of HSCs functions and prevent pro-apoptotic effects [3].

Cell therapy is currently a promising strategy for regenerative medicine, when cell types necessary for clinical needs begin to be used. Nevertheless, given the often medical inefficiency and the risk of complications from existing approaches, there is a need to develop new methods for cell application in a way aimed at restoring specific injuries. One of the new approaches to effective and directed changes in the properties of HSCs can be the use of contact interaction of MSCs and HSCs, which leads to significant activation of interacting cells [4].

Thus, the purpose of the study was to investigate the effect of transplantation of BMCs, intact and induced by the contact with MSCs, on regeneration of the immune system and hematopoiesis in mice after cyclophosphamide treatment, which in the future could be the basis for developing more effective methods to restore the immune system.

\section{MATERIALS AND METHODS}

Experiments were conducted on male C57BL/6 mice aged 6-8 weeks and weighing $18-20 \mathrm{~g}$ from the vivarium of Kavetsky Institute of Pathology, Oncology and Radiobiology of the National Academy of Sciences of Ukraine, which received a balanced diet and had free access to water ad libitum. All studies on experimental animals were carried out in compliance with the requirements of Article 26 of the Law of Ukraine «On the Protection of Animals from Cruelty» (February 21, 2006) and the European Convention for the Protection of Vertebrate Animals Used for Experimental and Other Scientific Purposes (Strasbourg, 1986)

To study the effect of cells on regeneration of the immune system of syngeneic mice, four groups were formed: I - normal animals, control $(n=10), I I$ - mice with cyclophosphamide treatment $(n=9)$, III - animals with cyclophosphamide treatment receiving BMCs $(n=7)$ and IV - with cyclophosphamide treatment receiving BMCs, induced by a previous 2-hour contact with MSCs (iBMCs) $(n=8)$.

After euthanasia of animals by cervical dislocation under ether anesthesia, thymuses and femurs, spleens and mesenteric lymph nodes were isolated under sterile conditions as well as peripheral blood was collected.

BMCs were obtained by flushing of the femoral diaphysis with a nutrient medium. Thymus-derived MSCs were obtained from C57BL/6 male mice at the age of 6-8 weeks by the standard explants technique [5]. Cultivation was carried out in DMEM/F12 (Sigma, USA), supplemented with $10 \%$ fetal bovine serum FBS (Sigma, USA), 10 mM L-glutamine (Sigma, USA) and $100 \mathrm{IU} / \mathrm{mL}$ penicillin and $100 \mu \mathrm{g} / \mathrm{mL}$ streptomycin (Darnytsya, Ukraine) in a $\mathrm{CO}_{2}$ incubator (Jouan, France) at $37{ }^{\circ} \mathrm{C}$ and $5 \%$ in the $\mathrm{CO}_{2}$ atmosphere. Subcultivation of cells in the ratio $1: 3$ was carried out using a mixture of $0.05 \%$ trypsin (Biotestmed, Ukraine) and $0.02 \%$ EDTA (Sigma, USA) solutions. The obtained cells were attached to plastic, had fibroblast-like morphology and formed colonies. The characteristics of MSCs were additionally established by inducing their differentiation into the osteogenic and adipogenic directions in the specific media $[6,7,8]$. Cells of $4-5^{\text {th }}$ passage were used for BMCs induction.

To model the immunodeficiency in mice of II, III and IV groups, single intraperitoneal injection of cyclophosphamide was administered at a standard dose $200 \mathrm{mg} / \mathrm{kg}$, animals of group I received saline. After 2 hours according to the metabolism of cyclophosphamide [9], $1 \cdot 10^{6}$ BMCs or
iBMCs in $0.1 \mathrm{~mL}$ of DMEM/F12 medium supplemented with $5 \%$ mouse serum were injected to the retroorbital sinus. Induced BMCs were obtained by incubation of BMCs on thymus-derived MSCs monolayer for 2 hours. Then BMCs, which detached from thymus-derived MSCs, were collected by light pipetting. In a week, mice of all groups were immunized intraperitoneally with $1 \cdot 10^{8}$ sheep erythrocytes. After 4 days, a repeated injection of the same number of cells subcutaneously into the footpad was performed, and after a day, the number of antibody-producing cells was measured by local hemolysis in gel, the delayed hypersensitivity rate by the difference in the mass of the experimental and control feets [10]. The control mice were immunized according to the same scheme. Thus, the study of the immune system of animals in all groups was carried out 12 days after the cyclophosphamide treatment, considering the dynamics of its immunosuppressive effect [11].

The number of erythrocytes, hematocrit, hemoglobin concentration in the peripheral blood was determined by the automatic hematologic anaIyzer Particle Counter PCE-210 (ERMA Inc., Japan). The analysis of the cell cycle and apoptosis of cells from mesenteric lymph nodes was performed by propidium iodide staining using FACScan flow cytometer (Becton Dickinson, USA) counting $25 \cdot 10^{3}$ cells per sample. ModFit LT software was used to establish the distribution of cells in the cell cycle phases [12].

The study of proliferative and cytotoxic activity of natural killer lymphocytes was performed by the Mossman method [13]. The phagocytosis activity of peritoneal macrophages with FITC-labeled S. aureus was investigated by a flow cytometry [14]. The level of spontaneous and induced bactericidal activity of peritoneal macrophages was determined by their ability to recover nitro blue tetrazolium (NBT) [15].

The obtained results are processed by the methods of variation statistics using MS Office Excel (Microsoft, USA) software. For quantitative parameters mean and standard error of mean $(\mathrm{M} \pm \mathrm{m})$ were calculated. The non-parametric Mann-Whitney U test was used to determine the significance of the differences between the compared groups. The critical value of the significance level was considered $p<0.05$.

\section{RESULTS AND DISCUSSION}

Twelve days after cyclophosphamide treatment, body weight of mice decreased by $13 \%(p<0.01)$ compared with the control group of intact animals, which is likely to be the result of the toxic effect of cyclophosphamide. After the injection of both BMCs and iBMCs into mice, the body weight was at the same level as of the animals treated with cyclophosphamide. Therefore, it can be assumed that the bone marrow cells did not have antitoxic effect.

The cyclophosphamide treatment resulted in a $36 \%$ reduction in bone marrow cellularity $(p<0.001)$ compared with the control group of intact animals. The introduction of BMCs caused even more pronounced cellularity reduction up to $53 \%(p<0.005)$ as compared with the control group (Fig. 1 A). After the injection of iBMCs, this index no longer had a significant difference from the norm, indicating the positive effect of these transplanted cells on the bone marrow recovery.

Reduction of bone marrow cellularity after the injection of BMCs following cyclophosphamide treatment was unexpected, because it would seem that BMCs should proliferate, differentiate and ensure the restoration of bone marrow cellularity that was observed after the introduction of iBMCs, which had a positive effect on the recovery of bone marrow.

Among the populations of hematopoietic cells, HSCs are uniquely able to migrate to the recipient bone marrow, as well as proliferate there and provide long-term hematopoiesis. In the bone marrow, HSCs are in specialized stromal niches, where their self-renewal and multipotency are provided [16]. However, irradiation and chemotherapy, which are used for both therapeutic purposes and for the conditioning of donor HSCs in the clinic, exhaust most of the hematopoietic system, including bone marrow niches [17]. We can assume that the transplanted normal BMCs do not reach the bone marrow niches, where they usually migrate, as the 


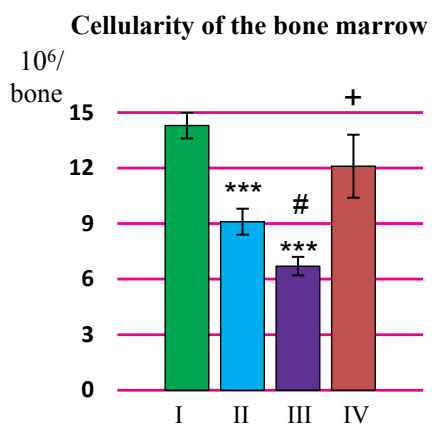

A

Cellularity of mesenteric lymph nodes

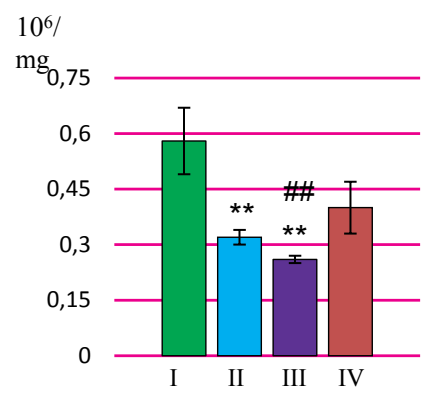

(D)

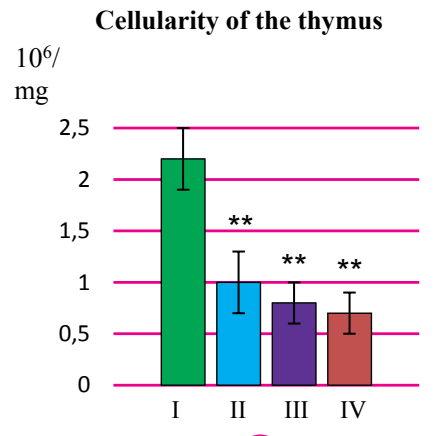

B

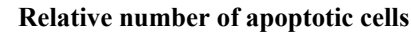
$\%$ in the mesenteric lymph nodes

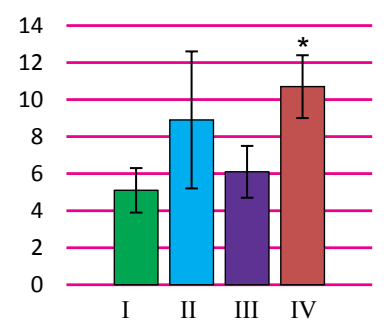

E

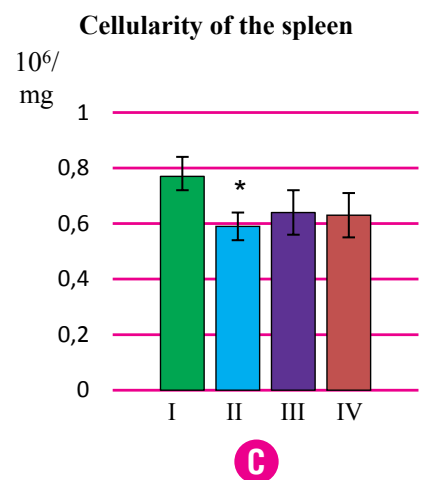

Relative number of cells of mesenteric lymph nodes in $\mathbf{G}_{\mathbf{0}} / \mathbf{G}_{\mathbf{1}}$ $\%$

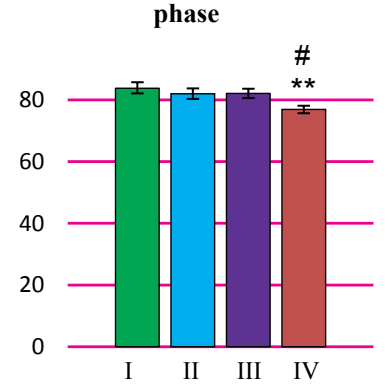

F
Relative number of cells of mesenteric lymph nodes in $\mathbf{S}+\mathbf{G}_{2} / \mathrm{M}$

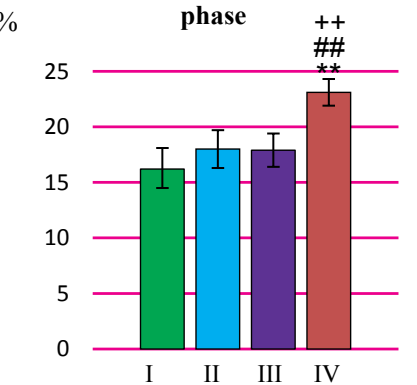

G

Fig. 1. Cellularity of bone marrow, lymphoid organs and proliferative activity of the murine cells: I - control animals; II - animals with cyclophosphamide treatment; III - animals with cyclophosphamide treatment receiving bone marrow cells; IV - animals with cyclophosphamide treatment receiving bone marrow cells induced by contact with thymus-derived multipotent stromal cells.

Notes: ${ }^{*}-p<0.05,{ }^{* *}-p<0.01,{ }^{* * *}-p<0.001$ - the difference is significant compared to the group of normal mice that received saline; $\#-p<$ 0.05 , \# $-p<0.01$ - compared to the group of mice with cyclophosphamide treatment; $+-p<0.05,++-p<0.01$ - compared to the group of mice with cyclophosphamide treatment that received bone marrow cells.

microenvironment in the niches suffers from the cyclophosphamide effect. As a result, BMCs do not demonstrate a complete restoration of the bone marrow in contrast to iBMCs that have been co-cultured with MSCs in vitro, and apparently, due to this, having got into the conditions of even exhausted bone marrow niches, they can fully develop, differentiate and restore the cellularity of bone marrow.

Under the impact of cyclophosphamide, the thymus weight reduced by $69,3 \%(p<0.005)$, total number of thymocytes reduced by $87,2 \%$ (p $<0.001)$ and the thymus cellularity reduced by $63,6 \%(p<0.01)$ compared to the control group of intact mice, which testified the involution of the thymus (Fig. 1 B). The transplantation of cells did not have an effect on these parameters. This is due to the fact that 12 days are not enough for their restoration because thymus-derived precursor cells are already exhausted and migration of precursors from bone marrow is disturbed both quantitatively and qualitatively [18].

The weight and the relative mass of the spleen in mice treated with cyclophosphamide were significantly increased after 12 days. At the same time, the number of splenocytes did not change and cellularity of the spleen even reduced. Characteristic change of these indexes allows to assume that the spleen enlargement under the impact of cyclophosphamide is the result of vascular disorders and edema of the lymphoid tissue. After the administration of BMCs into mice, spleen weigh and its cellularity normalized significantly. Injection of iBMCs led to a greater increase in the spleen weight, however, total number of splenocytes also increased. Therefore, cellularity did not differ from the one in normal mice (Fig. 1 C). Thus, these experimental conditions reveal the positive effect of bone marrow cells and more pronounced effect of induced BMCs on the spleen.

Treatment with cyclophosphamide reduced the cellularity of mesenteric lymph nodes by $44.8 \%(p<0.01)$ compared to the control group of intact mice. BMCs transplantation led to the greater reduction of this index by $55.2 \%(p<0.001)$ (Fig. 1 D). However, the injection of iBMCs completely restored the cellularity of the lymph nodes. Corresponding difference of the effect of cells transplantation has already been in the indexes of the bone marrow and once more demonstrates that contact interaction of MSCs and HSCs added new features to the HSCs.

The level of spontaneous apoptosis of lymph node cells under the impact of iBMCs increased significantly compared with the control group of intact mice (Fig. $1 \mathrm{E}$ ), which usually correlates with stimulation of cell proliferation in normal animals.

Data on the positive effects of iBMCs on lymph node cells and stimulation of proliferative activity are confirmed by the results of the study of the cell cycle phases. The number of cells in the $G_{0} / G_{1}$ phases has significantly reduced (Fig. $1 \mathbf{F}$ ), and in the $S+G_{2} / M$ phases has significantly increased (Fig. $1 \mathbf{G}$ ). While intact BMCs did not have such activity, it again indicates the divergence in the effects of the studied cells. There are studies in which a significant increase in the number of cells in the $G_{2} / M$ phase was observed in the non-adherent cells when MSCs and HSCs are co-cultured [19]. These cells were used in our experiment.

Thus, as a result of induced BMCs administration, but not intact BMCs, into mice treated with cyclophosphamide showed partial normalization of cellular parameters in various organs of the immune system. The efficacy of MSCs as an inductors of HSCs at bone marrow transplantation may be explained by the contact interaction of HSCs and stromal cells, as well as by the production of cytokines and growth factors necessary for homing and differentiation of HSCs [20,21].

The effect of transplantation of cells on red blood cells was the opposite, that is, intact BMCs were much more active in the regeneration of erythropoiesis. The treatment with cyclophosphamide significantly 
Total number of red blood cells in $10^{6} \%$ blood

$\mu \mathrm{L}$

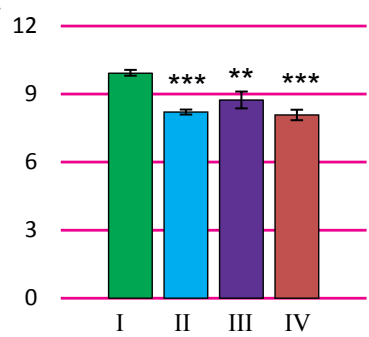

A
Relative number of reticulocytes $\%$

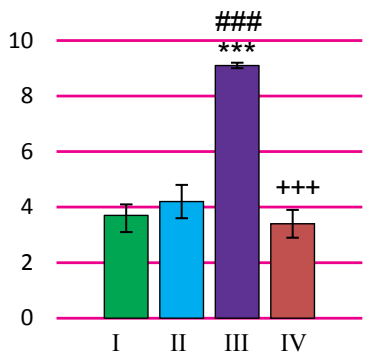

B
Hematocrit

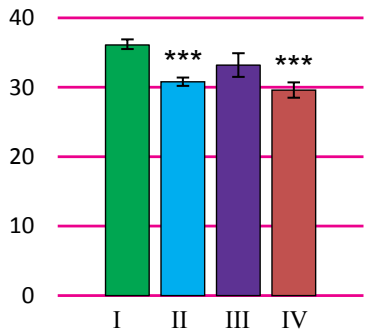

C

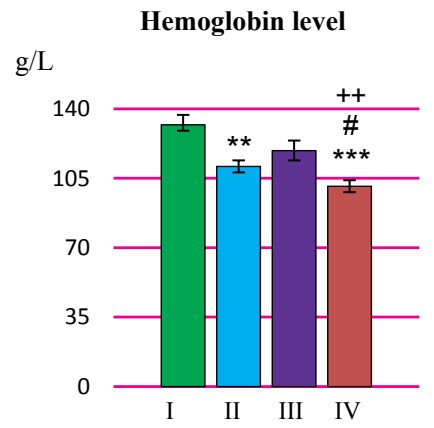

(D)

- Fig. 2. Hematological parameters of mice: I - control group; II - animals treated with cyclophosphamide; III - animals treated with cyclophosphamide and receiving bone marrow cells; IV - animals treated with cyclophosphamide and receiving bone marrow cells induced by contact with multipotent stromal cells.

Notes: ${ }^{*}-p<0.05,{ }^{* *}-p<0.01,{ }^{* *}$ - the difference is significant compared to the group of normal mice that received saline; \# $-p<0.05$, $\# \#$ - $p<0.001$ - compared to the group of mice with cyclophosphamide treatment; $++-p<0.01,+++-p<0.001-$ compared to the group of mice with cyclophosphamide treatment and transplantation of bone marrow cells.

\section{Bactericidal activity of peritoneal} macrophages

$\%$

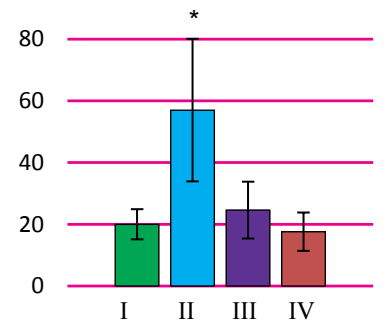

(A)

Number of antibody-producing cells in spleen

$/ 10^{6}$

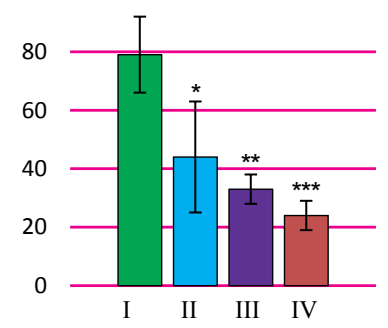

E

\section{Cytotoxic activity of splenocytes}

$\%$

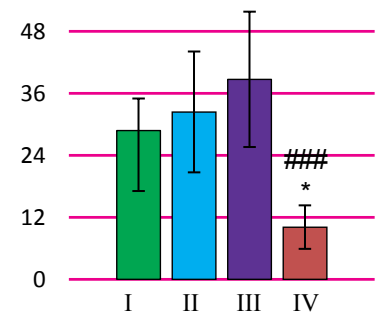

B

\section{Phytohemagglutinin-induced blast transformation of splenocytes}

$\%$

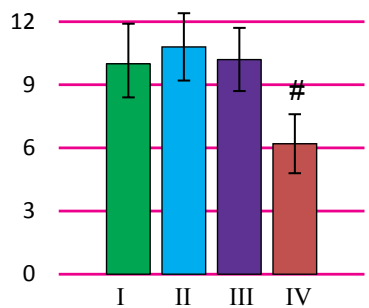

c
Delayed hypersensitivity response

$\%$

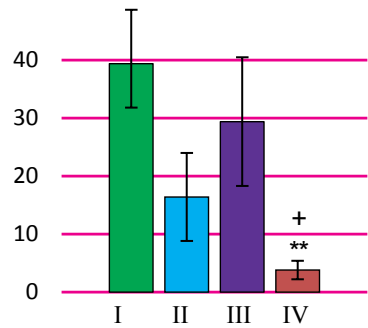

(D) reduced hematocrit, the number of red blood cells and hemoglobin. Transplanted iBMCs did not affect these parameters, while intact BMCs normalized hematocrit and hemoglobin and at the same time significantly increased the number of reticulocytes in the blood (Fig. 2).

The functional activity of the immune system underwent pronounced changes. If the absorption activity of macrophages did not change neither after the application of cells, nor because of the cyclophosphamide treatment. It is known that cyclophosphamide does not have suppressive effects on macrophages, and even vice versa, low doses of cyclophosphamide in oncology are used to enhance antigen-specific cellular immune response, as well as the activation of mechanisms of innate immunity mediated by the secretory function of macrophages [22]. A similar stimu- lating effect was observed when the bactericidal activity of peritoneal macrophages in the NBT-test after the cyclophosphamide treatment was substantially increased. This may also be due to the induction of bactericidal activity by products formed because of pro-apoptotic and toxic effects of cyclophosphamide on tissues, and may be due to the stimulatory action on phagocytes of conditionally pathogenic microflora activated by immunosuppressive effect of cyclophosphamide. Under the impact of transplanted cells, normalization of bactericidal activity was observed (Fig. 3 A), which most likely means that during 12 days, transplanted cells may contribute to the suppression of opportunistic infection.

Also, as a result of the introduction of iBMCs, there was a pronounced suppressive effect on the natural cytotoxicity of splenocytes (Fig. $\mathbf{3}$ B) and 
the blast transformation of splenocytes induced by phytohaemagglutinin (Fig. 3 C), which, after cyclophosphamide treatment and transplantation of BMCs, had no significant difference with control group. It is known that the application of cyclophosphamide has an immunomodulatory effect on the natural cytotoxicity of splenocytes, which may be both suppressing and stimulating depending on the conditions [23]. It is likely that in our experiment, the cyclophosphamide did not change of this parameter. Induced BMCs, acquiring new properties different from intact BMCs, are capable of suppressing cytotoxicity, the mechanism of such effects has not been studied, but is of interest for future researches.
These data correlate with the study of adaptive immunity, which showed the inhibitory effect of iBMCs on the delayed hypersensitivity response in mice (Fig. $\mathbf{3} \mathbf{D}$ ), which was normal in the use of intact BMCs. Meanwhile, the number of antibody-producing cells in the spleen of the mice treated with cyclophosphamide was significantly lowered, and none of the transplanted cell types had normalized its level (Fig. $3 \mathrm{E}$ ). The absence of effect of CD34+ hematopoietic cells transplantation on both cellular and humoral immune responses was also demonstrated on the humanized (RAG-hu) model of immunodeficient mice [24].

\section{CONCLUSION}

In general, the results indicate that, unlike normal cells, bone marrow cells induced by thymus-derived MSCs provide increased spontaneous proliferative activity of lymphocytes with the restoration of bone marrow, Iymphoid organs integrity, and also have a strong negative effect on natural cytotoxicity, delayed hypersensitivity response and number of antibody-producing cells. Normal BMCs provide regeneration of erythropoiesis. Probably, BMCs induced by thymus-derived MSCs lose this ability and are strongly activated in the direction of impact on the immune system. This is most likely due to the influence of contact interaction with thymus-derived MSCs, which play an important role in the functioning of thymus and have pronounced immunomodulatory properties.

\section{REFERENCES}

1. Morrison SJ, Scadden DT. The bone marrow niche for haematopoietic stem cells. Nature. 2014; 505(7483):327-34. DOI: 10.1038/nature12984.

2. Méndez-Ferrer S, Michurina TV, Ferraro F, Mazloom AR, et al. Mesenchymal and haematopoietic stem cells form a unique bone marrow niche. Nature. 2010; 466(7308):829-34. DOI: 10.1038/nature09262.

3. Block GJ, Ohkouchi S, Fung F, et al. Multipotent stromal cells are activated to reduce apoptosis in part by upregulation and secretion of stanniocalcin-1. Stem Cells. 2009; 27(3):670-681. DOl: 10.1002/stem.20080742.

4. Nikolskaya EI, Butenko GM. Structural-functional organisation of the bone marrow hematopoietic stem cells niches. Cell and organ transplantology. 2016; 4(1):82-100.

5. Prockop DJ, Phinney DG, Bunnell BA. Mesenchymal stem cells: methods and protocols. Humana Press. 2008. $192 \mathrm{p}$.

6. Gregory $C A$., Gunn WG, Peister A., et. al. An Alizarin red-based assay of mineralization by adherent cells in culture: comparison with cetylpyridinium chloride extraction. Anal Biochem. 2004; 329(1):77-84. DOI: 10.1016/j.ab.2004.02.002

7. Kim WK, Jung H, Kim DH, et al. Regulation of adipogenic differentiation by LAR tyrosine phosphatase in human mesenchymal stem cells and 3T3-L1 preadipocytes. J Cell Sci. 2009; 122(22):4160-7. DOl: 10.1242/jcs.053009.

8. Nikolskiy IS, Nikolskaya VV, Demchenko DL, Zubov DO. Potentiation of directed osteogenic differentiation of thymic multipotent stromal cells by prior co-cultivation with thymocytes. Cell and Organ Transplantology 2016; 4(2):220-223. DOI:10.22494/cot.v4i2.59.

9. Said R, Abdel-Rehim M, Sadeghi B, et al. cyclophosphamide Pharmacokinetics in Mice: A Comparison Between Retro Orbital Sampling Versus Serial Tail Vein Bleeding. The Open Pharmacology Journal. 2007; 1:30-35. DOl: 10.2174/1874143600701010030.

10. Frimmel G. Immunological methods edited by Frimel G. M: The world. 1987. 472 p.

11. Nikolsky IS. Dynamic features of hemo-immune deficiency induced by cyclophosphamide Proceedings of the international forum «Clinical immunology and allergology». 2014:195-196.

12. Khaitov RM, Pinegin BV, Yarilin AA. Guide to Clinical Immunology. Diagnosis of immune system diseases: a guide for doctors. M: GEOTAR-Media. 2002. $352 \mathrm{p}$.

13. Mossman T. Rapid colorimetric assay for cellular growth and survival application to proliferation and cytotoxicity assay. J Immunol Methods. 1983; 65(1-2):55-63.

14. Sugiura $H$, Nishida $H$, Inaba $R$, et al. Effects of different durations of exercise on macrophage functions in mice. J Appl Physiol. 2001; 90(3):789-94. DOI: 10.1152/ jappl.2001.90.3.789.

15. Zhang X, Goncalves R, Mosser DM. The isolation and characterization of murine macrophages. Curr Protoc Immunol. 2008; 14(1). D0I: 10.1002/0471142735. im1401s83.

16. Acar M, Kocherlakota K., Murphy S., et al. Deep imaging of bone marrow shows non-dividing stem cells are mainly perisinusoidal. Nature. 2015. 526, № 7571. P. 126-30. DOI: 10.1038/nature15250.

17. Abbuehl J-P, Tatarova Z, Held W, et al. Long-Term Engraftment of Primary Bone Marrow Stromal Cells Repairs Niche Damage and Improves Hematopoietic Stem Cell Transplantation. Cell Stem Cell. 2017. 21, № 2. P. 241-255. D0I: 10.1016/j.stem.2017.07.004. doi: 10.1016/j.stem.2017.07.004

18. Frasca D, Guidi F, Arbitrio $M$, et al. Hematopoietic reconstitution after lethal irradiation and bone marrow transplantation: effects of different hematopoietic cytokines on the recovery of thymus, spleen and blood cells. Bone Marrow Transplant. 2000; 25(4):427-33. DOI: 10.1038/sj.bmt.1702169.

19. Jing D, Fonseca AV, Alakel $\mathrm{N}$, et al. Hematopoietic stem cells in co-culture with mesenchymal stromal cells--modeling the niche compartments in vitro. Haematologica. 2010; 95(4):542-50.

20. Patel DM, Shah J, Srivastava AS. Therapeutic potential of mesenchymal stem cells in regenerative medicine. Stem Cells Int. 2013; 2013:496218. D0l: $10.1155 / 2013 / 496218$.

21. Rota C, Morigi M, Cerullo D, et. al. Therapeutic potential of stromal cells of non-renal or renal origin in experimental chronic kidney disease. Stem Cell Res Ther. 2018; 9(1):220. DOI: 10.1186/s13287-018-0960-8.

22. Bryniarski K, Szczepanik M, Ptak M, et. al. Influence of cyclophosphamide and its metabolic products on the activity of peritoneal macrophages in mice. Pharmacol Rep. 2009; 3:550-7. 
23. Teriukova NP, Pogodina ON, Blinova GI, Ivanov VA. Immunomodulating effect of cyclophosphamide on cytotoxic activity of rats and mice splenocytes. Tsitologiia. 2011; 53(10):800-7.

24. Tanner A, Hallam SJ, Nielsen SJ, et al. Development of human B cells and antibodies following human hematopoietic stem cell transplantation to Rag2(-/-)yc(-/-) mice. Transpl Immunol. 2015; 32(3):144-50. D0I: 10.1016/j.trim.2015.03.002.

年

The author indicates no potential conflicts of interest.

Received: September 18, 2018

Accepted: November 30, 2018 